\title{
Prediction of Call Drops in GSM Network using Artificial Neural Network
}

\author{
Olaonipekun Oluwafemi Erunkulu,1), Elizabeth Nnonye Onwuka ${ }^{2)}$, Okechukwu Ugweje ${ }^{3)}$, Lukman Adewale Ajao ${ }^{1)}$ \\ ${ }^{1)}$ Department of Computer Engineering, Federal University of Technology Minna \\ Main Campus, Gidan Kwanu, Along Minna - Bida Road; PMB 65 Minna, Niger State, Nigeria \\ ${ }^{2)}$ Department of Telecommunications Engineering, Federal University of Technology Minna \\ Main Campus, Gidan Kwanu, Along Minna - Bida Road; PMB 65 Minna, Niger State, Nigeria \\ ${ }^{3)}$ Department of Electrical and Electronics Engineering, Nigerian Turkish Nile University State \\ Cadastral Zone, Plot 681 Airport Rd, Jabi, Abuja, Nigeria
}

\begin{abstract}
How to Cite: O. O. Erunkulu, E. N. Onwuka, O. Ugweje, and L. A. Ajao, "Prediction of Call Drops in GSM Network using Artificial Neural Network," Jurnal Teknologi dan Sistem Komputer, vol. 7, no. 1, 2019. doi: 10.14710/jtsiskom.7.1.2019.38-46, [Online].
\end{abstract}

\begin{abstract}
Global System for Mobile communication is a digital mobile system that is widely used in the world. Over the years, the number of subscribers has tremendously increased, the quality of service (Call Drop Rate) became an issue to consider as many subscribers were not satisfied with the services rendered. In this paper, we present the Artificial Neural Network approach to predict call drop during an initiated call. GSM parameters data for the prediction were acquired using TEMS Investigations software. The measurements were carried out over a period of three months. Post analysis and training of the parameters was done using the Artificial Neural Network to have an output of " 0 " for no-drop calls and "1" for drop calls. The developed model has an accuracy of $87.5 \%$ prediction of drop call. The developed model is both useful to operators and end users for optimizing the network.
\end{abstract}

Keywords - Artificial Neural Network; call drop rate; Global System for Mobile communication; performance indicator; Quality of Service

\section{INTRODUCTION}

In the year 2001, the Global System for Mobile communication (GSM) was introduced to Nigeria and Nigerians embraced it with both hands. GSM network is a wireless communication system which was adopted to serve as an alternative to the analog system (Nigerian Telecommunications Limited). Its introduction was to provide effective telecommunication services which will support better speech quality, spectral efficiency, roaming, and minimize crosstalk [1]. GSM was a welcomed development that had changed the face of Information and Communications Technology in Nigeria. GSM has made a positive impact on the lives of Nigerians, enabling effective communication in the cities, and also in rural areas.

\footnotetext{
*) Correspondence author (E. O. Oluwafemi)

Email: femi.erunkulu@futminna.edu.ng
}

Before GSM Network was deployed in the Nigerian market, access to the telephone was a luxury which was meant for some few people. Only four hundred and fifty thousand working telephone lines were available as early 2001. The number of GSM subscribers had been on the increase since the invention of the network. With this increase and without a corresponding increase in resources, the quality of the service began to fall. For a GSM network operator to retain and likewise expand the base of her subscriber, the subscribers' satisfaction with the delivered user-perceived Quality of Service (QoS) is their main objective. Performance evaluation, traffic analysis, and optimization are therefore important for network planning, and design [2]. Therefore, it became an important problem to be considered because many subscribers' satisfaction with the rendered services had been low.

All over the world as well as in Nigeria, poor QoS has been a major problem over the past years. QoS in mobile networks shows the capability of the mobile service operators to render acceptable services, which include low call blocking, clear voice quality, high signal strength, low call drop as well as high data rates for data and multimedia applications [3]. Poor QoS has been the order of the day for subscribers, ranging from unceasing call drops, congestion in network, bad voice clarity and paradox in the billing system. With all these factors, most subscribers were forced to purchase dual SIM slots phones. A good number of subscribers in Nigeria own two to four cell phones. Many GSM subscribers are disgruntled with the persistent poor telecom services.

Due to so many reasons, operators find it tasking to deliver acceptable QoS to subscribers. According to the Nigerian Communications Commission (NCC), active GSM subscribers in Nigeria is 149,787,120 million that is about $80.5 \%$ of the Nigerian population [4]. However, the QoS provided by the GSM operators in Nigeria has remained abysmal and every subscriber is affected. In order to enforce better service quality, the NCC set a benchmark for the key performance indicators (KPIs) for every GSM operator. 


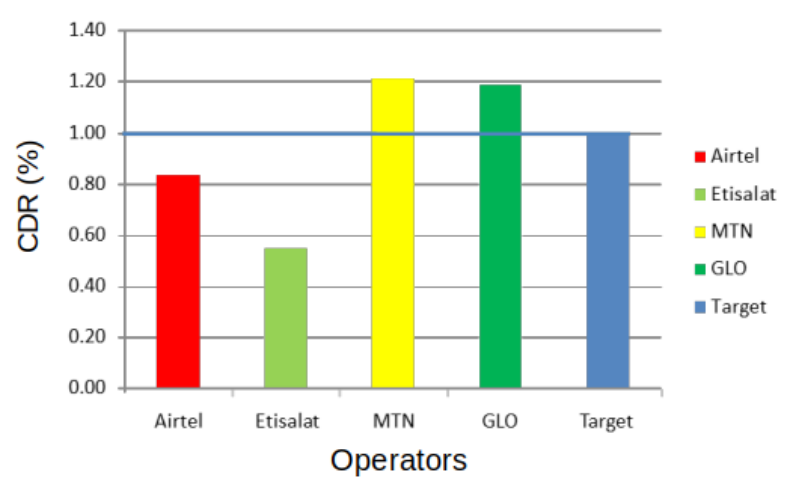

Figure 1. The result of Call Drop Rate test showing the threshold target [5]

The KPIs metrics are used for measuring the QoS of the GSM networks [5]-[7]. These standards define the lower and upper bounds of acceptability. Some of these include call drop rate (CDR), call set-up success rates (CSSR), call completion rates (CCR), handover success rate (HSR), and traffic channel congestion rate (TCHCR), Transmission rates, among others. These QoS standards ensure the subscribers' continuity to access the desired network with better quality telecommunications service. The governing body sets the basic minimum quality levels for all operators [8], [9]. The parameters used in measuring GSM QoS are network accessibility, network retainability, connection quality, and charging accuracy. The first three of these parameters are used in assessing the performance of the networks which are subjected to frequent changes with respect to increase coverage and capacity demands [10].

Call drop rate (CDR) is one of the KPIs. According to NCC, $1 \%$ was set as the threshold target for all operators. Calls are dropped if a mobile subscriber moves out of a coverage or serving area and the signal cannot be maintained between the phone and the network. The call could drop if the received signal level falls to about -95 to $-110 \mathrm{dBm}$ due to path loss or shadowing. The result of a survey on CDR carried out by the NCC in January 2014 on all the GSM operators in Nigeria is shown in Figure 1 [4]. As the call drop rate increased tremendously, it becomes necessary to find a way to reduce the rate in order to increase the QoS of the mobile network.

Many research works have been carried out on measurements and prediction of call drop and how to improve such in GSM. However, these were done mostly using probability with different scenarios [11][14]. These predictions were based on acquired data from servers that had recorded dropped call events over periods of time. Another researcher collected the TCH (traffic channel) call drop events from a live GSM network and used ANN to predict the probability of drop call [15].

In this paper, we present the prediction of call drops in a real-time scenario, taking into account some GSM parameters using Artificial Neural Network (ANN). Data used in the call drop prediction model are five

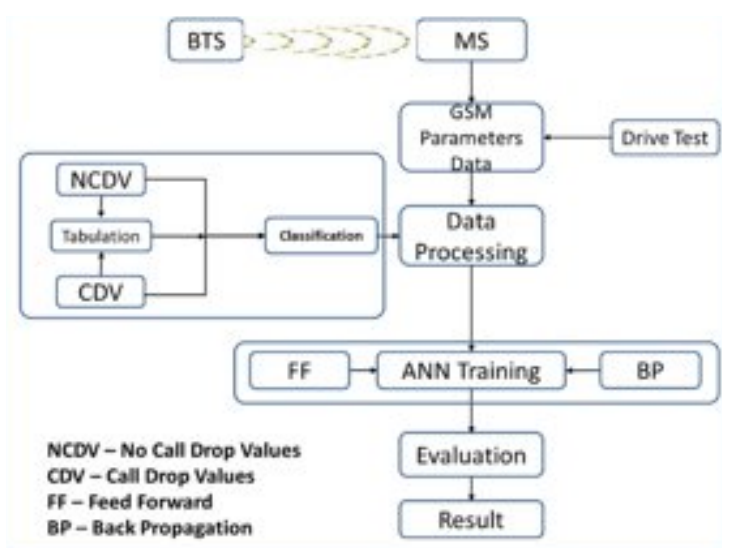

Figure 2. Simulation model schematic

GSM QoS parameters, namely Received Signal Level (RxLev), Received Quality (RxQual), Frame Error Rate (FER), Bit Error Rate (BER), and Timing Advance. The development of a call drop prediction model would be useful for operators to carry out a survey and thereby, optimizing their network to reduce CDR. We used a software package called TEMS Investigation installed on a laptop. Also, three mobile stations (MS) were connected to the laptop to acquire the data. Drive testing was the method used in the city of Abuja, Nigeria for a period of three months.

\section{RESEARCH METHODS}

The method adopted to achieve prediction of call drops in GSM network using ANN in this research includes acquisition of data (data capturing), data processing, model development, performance evaluation, and call drop prediction. Figure 2 shows the schematic simulation stages involved in the proposed method. The CDR is defined as the number of dropped calls divided by the total number of call attempts as expressed in Eq. 1. It also be defined as (1-Call Completion Ratio) x 100\%.

$$
\text { CDR }=\frac{\text { Number dropped call }}{\text { Total number of call attempts }} * 100 \%
$$

\section{A. Data Capturing}

In order to acquire sufficient amount of data to predict the possibility of a call drop in an ongoing call, a Drive Test (DT) was performed to evaluate the QoS of a mobile network by assessing the coverage as well as the capacity of the network [16]. Mobile radio network measuring devices are installed in a motor vehicle. This device is capable of measuring and recording a wide variety of both physical and virtual parameters of geographical areas.

To perform the DT, a software package called TEMs Investigation was used for the measurement of the GSM services. It was used for verification, troubleshooting, maintenance, and optimization standard. The GSM parameters collected for the prediction of drop calls are 


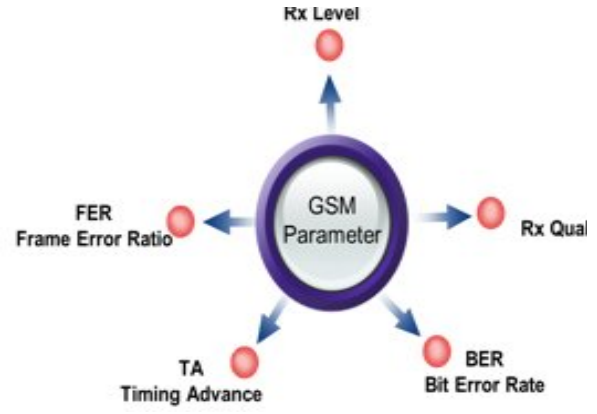

Figure 3. GSM parameters

shown in Figure 3. This tool is used to capture each of the specified parameters either in idle mode or dedicated mode and stored as log files. The drive tests (DT) was carried out over a period of three months in the city of Abuja, Nigeria.

\section{Received signal Level (RxLev)}

RxLev refers to the radio signal level or strength of the MS signal which was received from a base station transceiver's transmitting antenna. Specifically, RxLev is the signal strength (measured in $\mathrm{dBm}$ ) received by a MS from a BTS (on the downlink).

The RxLev of MS can be affected by factors such as obstructions, refraction, signal path loss and proximity to a BTS which can be calculated as expressed in Eq. 2. This signal level ranges from $-110 \mathrm{dBm}$ to $-47 \mathrm{dBm}$ as presented in Table 1 . The MS signal is stronger from $-47 \mathrm{dBm}$ and very low as it approaches $-110 \mathrm{dBm}$. The RxLev play significant roles in sustaining a connected call, the stronger the RxLev signal, the lesser the possibility of call drop.

$$
R x \operatorname{Lev}(d B m)=\operatorname{EiRP}(d B m)-\operatorname{Path} \operatorname{Loss}(d B)
$$

\section{Received Quality (RxQual)}

Based on BER analysis, RxQual is a measure of speech quality and used for speech quality evaluation in GSM network by transforming BER. This is computed from the average BER over a period of time, just before decoding the channel. Low degradation of speech quality occurs in rural cells, while in the case of urban areas, due to high interference, the poor QoS in communication is higher. Table 1 shows correlation of RxQual value with the estimated BER [17].

The RxQual can be used for controlling the downlink power, dynamic coding scheme adaptation and for network-controlled cell reselection. RxQual is at its best quality at scale 0 while, 7 is the worst. Each of the RxQual value correlates with an approximated number of bit errors in a given number of bursts.

\section{Frame Error Rate (FER)}

FER is used to determine the quality of a signal connection. It is a value between 0 and $100 \%$. The Slow Associated Control Channel (SACCH) multiframe is the base for all measurements, because, during one such
Tabel 1. BER to RxQual conversion [17]

\begin{tabular}{ccc}
\hline No & RxQual & Bit Error Rate (BER) \\
\hline 1 & 0 & BER $<0.2 \%$ \\
2 & 1 & $0.2 \%<\mathrm{BER}<0.4 \%$ \\
3 & 2 & $0.4 \%<\mathrm{BER}<0.8 \%$ \\
4 & 3 & $0.8 \%<\mathrm{BER}<1.6 \%$ \\
5 & 4 & $1.6 \%<\mathrm{BER}<3.2 \%$ \\
6 & 5 & $3.2 \%<\mathrm{BER}<6.4 \%$ \\
7 & 6 & $6.4 \%<\mathrm{BER}<12.8 \%$ \\
8 & 7 & $12.8 \%<\mathrm{BER}$ \\
\hline
\end{tabular}

frame, one SACCH message is transmitted from the BS to the MS and vice verse. A GSM TDMA frame is built up using eight consecutive timeslots, numbered 0-7 and a speech call will be assigned one of the eight timeslots.

The continuous stream of TDMA frames is formed by repeating the eight timeslots, where each MS is assigned a timeslot during a speech conversation. The GSM multiframe has 26 frames known as traffic multiframe, composed of 26 bursts in a duration of $120 \mathrm{~ms}$ that is, 26 consecutive timeslots assigned to an MS, 24 out of the 26 timeslots are used for transmitting the speech. The remaining two timeslots are used for signaling (SACCH) and identification of neighboring cells [18]. Where the FER is too high (many errors), call drop may occur. FER expression is shown in Eq. 3.

$$
F E R=\frac{\text { Data recieved } \text { with Error }}{\text { Total } \text { Data recieved }}
$$

\section{Bit Error Rate (BER)}

BER is the bits with errors divided by the total number of transmitted bits defined in percentage as shown in Eq. 4. This bit received is processed over a given period of time. This value indicates the number of bits the MS has received with an error.

$$
B E R=\frac{\text { Number of bit errors }}{\text { Total number of transferred bits }} * 100 \%
$$

At $-104 \mathrm{dBm}$, the maximum acceptable BER is $3 \%$. When this value is too high, some parts of the communication or control commands of the BTS could be lost and the communications may break up or the call will drop altogether [19]. In a mobile system, if the signal to noise ratio (SNR) is relatively high and the medium between the receiver and transmitter is good, then the BER will be negligible with no conspicuous effect. However, with the detection of noise, BER will be considered. BER is due to noise and changes in the propagation path where radio signal paths are used [20]. Problems with bit synchronization, interference, multipath fading in wireless, transmission channel noise, attenuation, and distortion may affect the MS.

\section{Timing Advance (TA)}

TA refers to the time length taken for an MS signal to communicate with the base station. In GSM, sharing of frequency among several users is achieved by TDMA 
technology by assigning sequential timeslots to different users sharing the same frequency.

The propagation delay in the signal as it travels between the MS and BTS is compensated by the TA. TA is assigned by the BSS (base station subsystem) to the MS based on how far away it perceives the MS to be. TA can be varied from 0 to 63 [21]. Since eight timeslots make a frame, an MS periodically transmits within the frame in less than $1 / 8^{\text {th }}$ of the time. Since the MS are located at different distances from the BTS and also signals travel at the speed of light, the BTS determines the distance position of the MS by using the arrival-time within the slot.

Collision prevention by adjacent MS is achieved by adjusting accordingly, the time permitted to each adjacent MS to transmit a burst of traffic within a timeslot. TA is the variable that controls this adjustment. Interference can be avoided to and fro other MS in adjacent timeslots by continual adjustment of TA value, with this, data loss is minimized and mobile QoS (call quality-of-service) is maintained. Figure 4 shows how the TEM Investigations platform is laid out, showing the parameter values. The two events when a call dropped and the cases where it did not drop were recorded.

\section{B. Pre-processing}

The event log files contain raw data. Each of the log files of different events was extracted and ran in order to extract the needed GSM parameter values. ANN gives better performance when input variables are preprocessed before being used for training. The five GSM parameter values were tabulated and read by MATLAB in the form of comma separated values (CSV).

\section{Input Data}

Drop-call with its corresponding drop-call target and no-drop-call with the corresponding target. The corresponding target for drop-call is one (1) while that of the no-drop-call target is zero (0).

\section{Training Data}

Data were extracted from the drop-call and no-dropcall data values which were classified as the training data and the corresponding training Target. These sets of data were used for neural network training.

\section{Validation Data}

For validating the neural network model, a set of test data and the corresponding target were also classified. All data were presented as Excel sheets.

\section{The Training Process}

The proposed neural network model in this work was developed by first training the network using the data sets collected from TEMS investigation and preprocessed as the inputs. Since the Neural network (NN)
DROPPED CALL

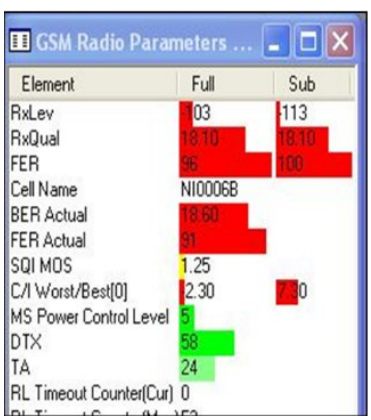

Figure 4. GSM parameter values GUI

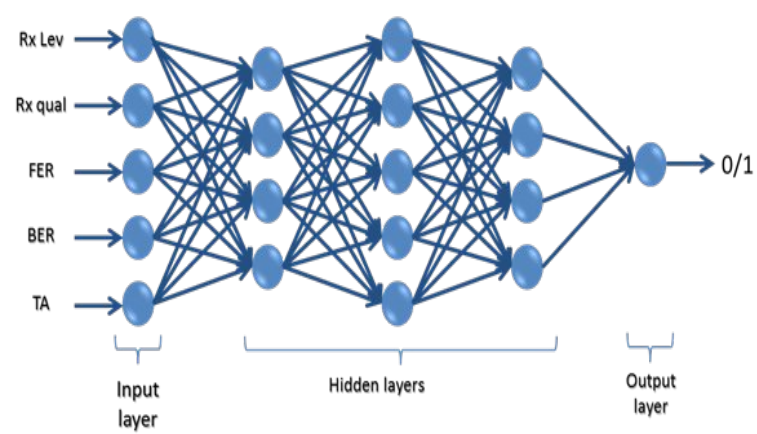

Figure 5. Deployed FFNN

can learn and therefore can be trained to find solutions, recognize patterns, and predict data.

This model used the feed-forward neural network (FFNN), a type of supervised NN. Figure 5 illustrates the neurons arrangement of FFNN. The inputs and outputs are real values. Each neuron was connected to each of the neurons in the previous layer and the strength of the coupling connection is determined by each weight. The input neurons are connected to hidden neurons, which perform transformations on the input. The output of these neurons is defined to be the output of the entire network.

In the training process, back-propagation algorithm was employed as illustrated by the flowchart of Figure 6 [22]. The training dataset was used to train the neural network. The network was trained by presenting the inputs and target outputs corresponding to the inputs. The error between the target and actual output in the learning algorithm was used to adjust the weights of the network until the desired output was obtained.

Each neuron is composed of two units. The first unit adds products of weight coefficients and input signals. The second unit realizes nonlinear function, called neuron transfer (activation) function. Signal $e$ is an adder output signal, and $y=f(e)$ is the output signal of the nonlinear element as illustrated in Figure 7 . Signal $y$ is also output signal of neuron $z$.

The training dataset consists of input signals $\left(x_{1}, x_{2}, x_{3}, x_{4}\right.$ and $\left.x_{5}\right)$ assigned to corresponding targets (desired output). Each training step starts with forcing both input signals from the training set. After this stage, 


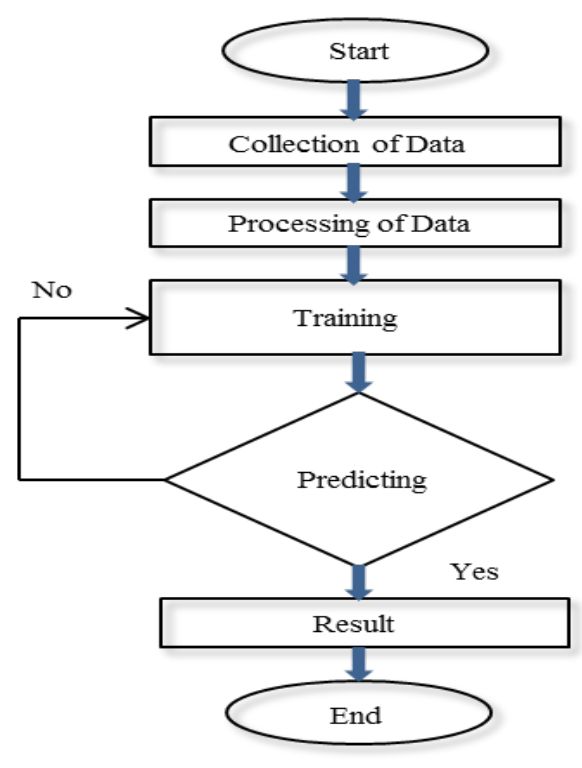

Figure 6. Drop call prediction flowchart

the output signal values for each neuron in each network layer was determined.

The network training is an iterative process. In each iteration weights coefficients of nodes are modified using new data from the training data set. Modification is calculated using the algorithm as expressed in Eq. 5 and Eq. 6.

$$
\begin{aligned}
& e=x_{1} w_{1}+x_{2} w_{2}+x_{3} w_{3}+x_{4} w_{4}+x_{5} w_{5} \\
y= & f(e) \\
= & f\left(x_{1} w_{1}+x_{2} w_{2}+x_{3} w_{3}+x_{4} w_{4}+x_{5} w_{5}\right)
\end{aligned}
$$

For each of the neurons in the network, there are five inputs. Symbols $w_{(x m) n}$ represent the weights of connections between network input $x_{m}$ and neuron $n$ in the input layer (Figure 8). Symbol $y_{m}$ represents output signal of neuron $n$. For the first column of the neuron with five inputs is expressed in Eq. 7. For the next column is expressed in Eq. 8.

$$
\begin{gathered}
y_{1}=f_{1}\left(x_{1} w_{(x 1) 1}+x_{2} w_{(x 2) 1}+x_{3} w_{(x 3) 1}+x_{4} w_{(x 4) 1}+x_{5} w_{(x 5) 1}\right) \\
y_{2}=f_{2}\left(x_{1} w_{(x 1) 2}+x_{2} w_{(x 2) 2}+x_{3} w_{(x 3) 2}+x_{4} w_{(x 4) 2}+x_{5} w_{(x 5) 2}\right) \\
\vdots \\
y_{4}=f_{4}\left(x_{1} w_{(x 1) 4}+x_{2} w_{(x 2) 4}+x_{3} w_{(x 3) 4}+x_{4} w_{(x 4) 4}+x_{5} w_{(x 5) 4}\right) \\
y_{5}=f_{5}\left(y_{1} w_{15}+y_{2} w_{25}+y_{3} w_{35}+y_{4} w_{45}\right) \\
\vdots \\
y_{9}=f_{9}\left(y_{1} w_{19}+y_{2} w_{29}+y_{3} w_{39}+y_{4} w_{49}\right)
\end{gathered}
$$

For simplicity, let $y_{10}=y_{a}, y_{13}=y_{d}, y_{14}=y_{t}$ and likewise the corresponding $w$, then neurons in the last column can be expressed in Eq. 9, and the output of the network is in Eq. 10.

$$
\begin{aligned}
& y_{a}=f_{10}\left(y_{5} w_{5 a}+y_{6} w_{6 a}+y_{7} w_{7 a}+y_{8} w_{8 a}+y_{9 a}\right) \\
& \vdots \\
& y_{d}=f_{13}\left(y_{5} w_{5 d}+y_{6} w_{6 d}+y_{7} w_{7 d}+y_{8} w_{8 d}+y_{9 d}\right)
\end{aligned}
$$

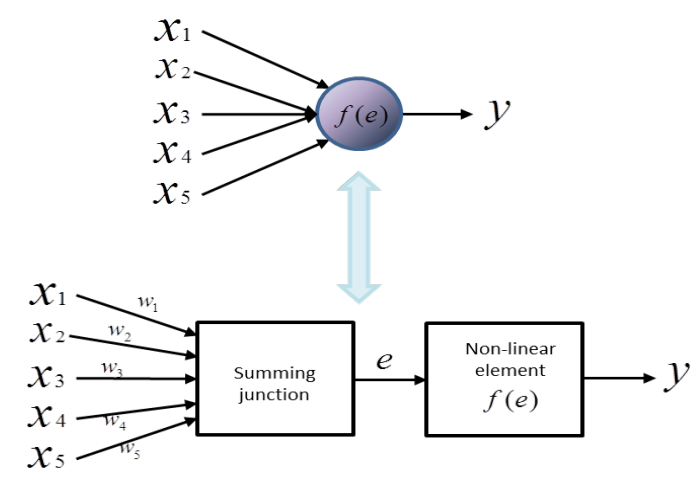

Figure 7. FFNN block diagram

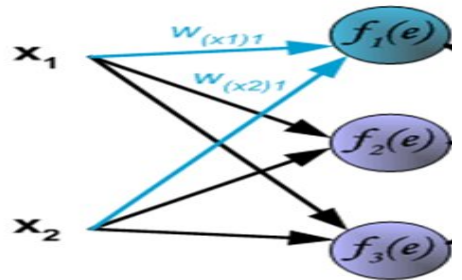

Figure 8. Signal propagation through neural network

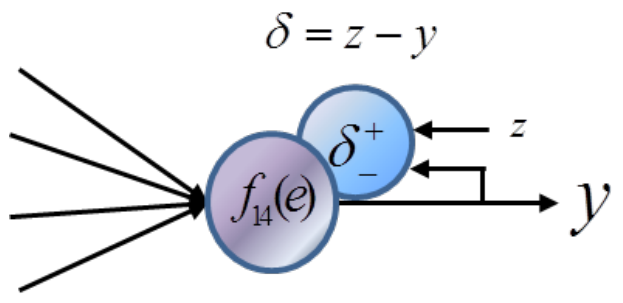

Figure 9. FFNN signal propagation values comparison

$$
y=y_{t}=f_{14}\left(y_{a} w_{a t}+y_{b} w_{b t}+y_{c} w_{c t}+y_{d} w_{d t}\right)
$$

In the next algorithm step, the output signal of the network $y$ is compared with the desired output value (the target), which is found in the training data set. The difference is called error signal $\delta$ of output layer neuron. Propagating the error signal $\delta$ back to all neurons (Figure 9), starting from the output signals is expressed in Eq. 11.

$$
\delta_{a}=w_{a t} \delta, \quad \delta_{b}=w_{b t} \delta
$$

The weights' coefficients $w_{m n}$ used to propagate errors back are equal to those used during computing output value. Only the direction of data flow is changed (signals are propagated from output to input one after the other). This technique is used for all network layers. If propagated errors came from a few neurons, they are added and expressed in Eq. 12 and Eq. 13.

$$
\begin{gathered}
\delta_{5}=w_{5 a} \delta_{a}+w_{5 b} \delta_{b}+w_{5 c} \delta_{c}+w_{5 d} \delta_{d} \\
\delta_{1}=w_{15} \delta_{5}+w_{16} \delta_{6}+w_{17} \delta_{7}+w_{18} \delta_{8}+w_{19} \delta_{9}
\end{gathered}
$$


When the error signal for each neuron is computed, the weight coefficients of each neuron input node may be modified. In Eq. 14 and Eq. 15, the $d f(e) / d e$ represents a derivative of neuron activation function (which weights are modified). Therefore, the general modification of weights is expressed in Eq. 16.

$$
\begin{gathered}
w_{(x 1) 1}^{\prime}=w_{(x 1) 1}+\eta \delta_{1} \frac{d f_{1}(e)}{d e} x_{1} \\
w_{56}^{\prime}=w_{56}+\eta \delta \frac{d f_{6}(e)}{d e} y_{5} \\
w_{m n}^{\prime}=w_{m n}+\eta \delta_{n} \frac{d f_{1}(e)}{d e} y_{m}
\end{gathered}
$$

A key feature of neural networks is an iterative learning process in which data cases (rows) are presented to the network one at a time, and the weights associated with the input values are adjusted each time. After all cases are presented, the process often starts over again. During this learning phase, the network learns by adjusting the weights so as to be able to predict the correct class label of input samples. The network processes the records in the training data one at a time using the weights and functions in the hidden layers, and then compares the resulting outputs against the desired outputs.

Errors are then propagated back through the system, causing the system to adjust the weights for application to the next record to be processed. This process occurs over and over as the weights are continually tweaked. During the training of a network the same set of data is processed many times as the connection weights are continually refined. We employed 5000 iterations for each run.

\section{RESULTS AND DISCUSSION}

Figure 10 shows one of the MS event plots in Abuja, as we took a drive test using TEMS Investigation software. This revealed where events such as drop calls and handover failures occurred on the route. The different colour of the route shows the signal strength. At each event of drop call, the reading of the five GSM parameters values was taken.

The model reads all the present GSM parameter values of the connected MS and could predict the possibility of a call dropping. At each point on the network, MS connected to a BTS has all this reading either in the idle or dedicated mode. As the MS moves from one location to another, signals are being sent to and fro the BTSs, the MS are handed over to another BTS when the GSM Parameter values are approaching a certain level. In other words, thresholds are used to set the cell location. In one of the drive test event, we noticed that an MS undergo handover from one BTS to another BTS when the signal (RxLev) is -80dbm.

In this study, it was discovered that drop call can occur even when the RxLev is very good. Other GSM parameters listed here add up to it. Table 2 and Figure

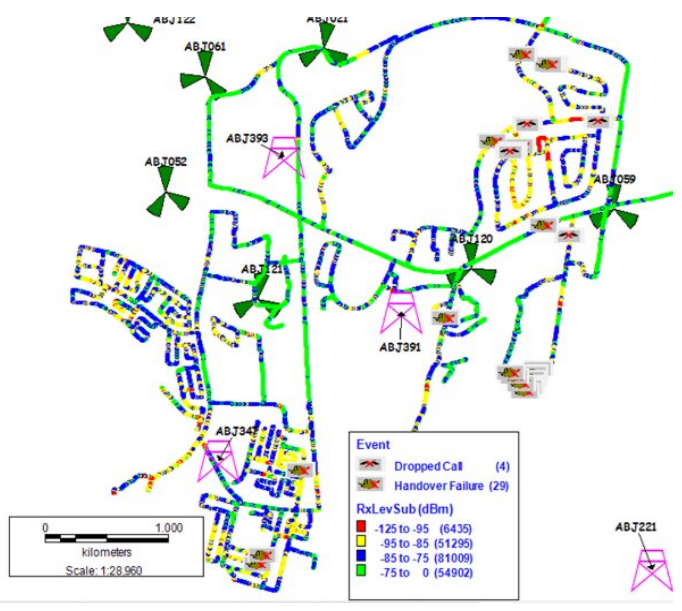

Figure 10. Mobile station drive test event map

Table 2. Prediction Table

\begin{tabular}{ccclccc}
\hline $\begin{array}{c}\text { RxLev } \\
(\mathrm{dBm})\end{array}$ & RxQual & FER & BER & TA & $\begin{array}{c}\text { Expected } \\
\text { Value }\end{array}$ & Prediction \\
\hline-103 & 18.1 & 100 & 8.9 & 1 & 1 & 0.6615 \\
-107 & 18.1 & 100 & 17.7 & 0 & 1 & 0.6615 \\
-92 & 18.1 & 100 & 25.2 & 5 & 1 & 0.6615 \\
-92 & 4.53 & 100 & 19.3 & 14 & 1 & 0.6615 \\
-108 & 18.1 & 100 & 17.9 & 23 & 1 & 0.6615 \\
-103 & 18.1 & 100 & 19.5 & 1 & 1 & 0.6615 \\
-98 & 9.05 & 100 & 13.7 & 7 & 0 & 0.3612 \\
-99 & 9 & 0 & 8.6 & 7 & 0 & 0.3612 \\
-93 & 9.05 & 0 & 16.2 & 4 & 0 & 0.3612 \\
-93 & 18.1 & 50 & 14.8 & 4 & 0 & 0.3628 \\
-96 & 9.05 & 0 & 16.7 & 4 & 0 & 0.3612 \\
-105 & 9.05 & 0 & 2.7 & 3 & 0 & 0.3612 \\
-106 & 9.05 & 0 & 10.6 & 3 & 0 & 0.3612 \\
-101 & 4.53 & 100 & 16.5 & 3 & 0 & 0.6615 \\
-108 & 18.1 & 100 & 19.2 & 23 & 0 & 0.6615 \\
-99 & 9.05 & 0 & 4.1 & 6 & 0 & 0.3612 \\
\hline
\end{tabular}

11, gives an idea of some of the present parameters reading of MS. The first six rows in Table 2 show the GSM parameter values (RxLev, RxQual, FER, BER, and TA respectively) of call drop. The expected value (column six) for the call drop was tagged as 1; the last column shows the prediction of the developed model. Notice that the predicted values were 0.6615 which is greater than the threshold of 0.5 . The model was designed to use the trained data along with the test data set to validate the accuracy of the model.

For the prediction, any predicted value that is equal to or greater than 0.5 is expressed as 1 , while less than 0.5 is expressed as 0 . The model was able to predict the call drop correctly. In the case of no-call-drop parameter values tested, the model prediction value was 0.3612 (which is lesser than 0.5), while that of call drop was 0.6615 . The developed model has an accuracy of $87.5 \%$ prediction of drop call.

Figure 11 shows the pattern of call drop values. These values were from the six GSM parameters used for testing the model. From across the range of six values, it was observed that the RxLev was in the range of -92 to $-107 \mathrm{dBm}$. This means that the signal strength 


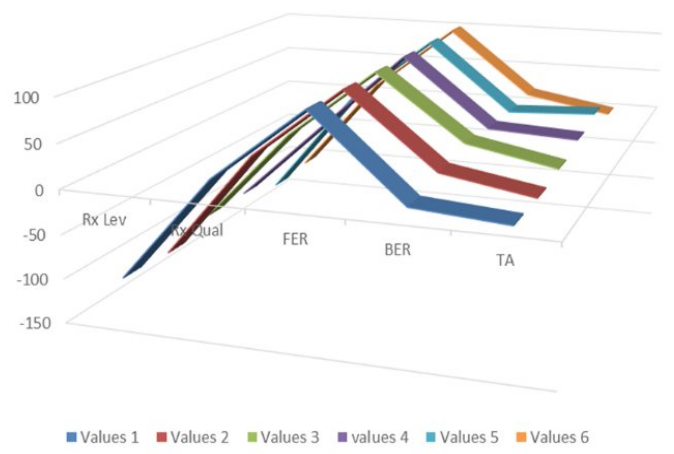

Figure 11. Illustration of the call drop parameters pattern

was always low. The RxQual is also poor, that is, above 0 . In the real scaling for RxQual $(0-7)$, the value closer to zero is better than that of seven. The FER is at its peak value of 100 for the six sets of GSM parameter values. BER is also high. This means error rate is high in the network. Lastly, the TA varies. It is moderate in some instants and high in others.

Figure 12 shows the parameter pattern of five nocall-drop events. For this study, we took parameter values that are high and close to the call drop values in order to have a better prediction model. The five RxLev values show different values but mostly above $-100 \mathrm{dBm}$. Though we have values around -101 to -106 . The FER had different values such as 0,50 , unlike the case of drop call values, where all the values were 100 . BER values varied. They were not as high as that of Figure 11. This means error rate is low in the network. Lastly, the TA values are very low compared to the ones in Figure 11.

Table 3 shows the parameter values of events where the call did not drop. The probability of call dropping with this parameter value is very low except in cases where network programme error occurred. The RxLev is very high starting from $-60 \mathrm{dBm}$. Not only the RxLev is high, other parameter values are good. For instance, the FER had zero value all throughout. The other parameters were just a bit above the zero values.

Looking at Table 4 and Table 5, it can be seen that, despite the fact that, RxLev values of Table 5 were very low, the call did not drop, unlike that of Table 4 . But the differences are shown in the other parameter values. In Table 4, the values of RxQual, FER and BER are higher, thereby forcing the MS to disconnect from the network. This shows that low RxLev is not enough to drop a call in some cases, but other parameters contribute to call dropping.

In this study, the call drop analysis of GSM operator networks was carried out. Also, a model for predicting drop call in an on-going call was developed using extracted data from a drive test using TEMS Investigations software. The use of ANN for the development of the model in this work has the advantage of training any network and prediction as long as correct data were supplied. The paper described a good approach for call drops detection as in [11]-[15]

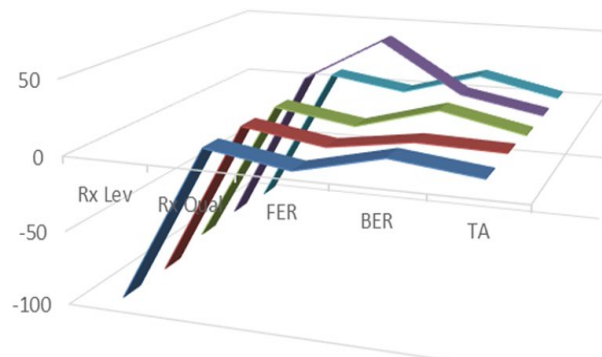

-Values 1 Values 2 Values 3 Values 4 Values 5

Figure 12. Illustration of no-call-drop parameter pattern

Table 3. No-call-drop values

\begin{tabular}{ccccc}
\hline RxLev $(\mathrm{dBm})$ & RxQual & FER & BER & TA \\
\hline-78 & 4.53 & 0 & 2.8 & 1 \\
-74 & 0.14 & 0 & 4 & 1 \\
-85 & 0.14 & 0 & 0 & 2 \\
-60 & 0.14 & 0 & 0 & 0 \\
-92 & 0.57 & 0 & 2.7 & 0 \\
-92 & 18.1 & 0 & 3 & 1 \\
-73 & 14 & 0 & 0 & 0 \\
\hline
\end{tabular}

Table 4. Call drop for GSM parameters values

\begin{tabular}{ccccc}
\hline RxLev $(\mathrm{dBm})$ & RxQual & FER & BER & TA \\
\hline-103 & 18.1 & 100 & 8.9 & 1 \\
-107 & 18.1 & 100 & 17.7 & 0 \\
-92 & 18.1 & 100 & 25.2 & 5 \\
\hline
\end{tabular}

Table 5. No-call-drop for GSM parameter values

\begin{tabular}{ccccc} 
RxLev $(\mathrm{dBm})$ & RxQual & FER & BER & TA \\
\hline-96 & 9.05 & 0 & 16.7 & 4 \\
-105 & 9.05 & 0 & 2.7 & 3 \\
-106 & 9.05 & 0 & 10.6 & 3
\end{tabular}

by using different scenarios. It has been demonstrated that a classification of call drop events can be made with this approach based on five GSM parameters, namely RxLev, RxQual, FER, BER and TA. These parameters contribute to calls dropping and reduces the Quality of Service (QoS). While in most related research used probability to determine call drop rate in different networks [11]-[14] and another focuses on RxLev, handover failure, and low power status of Mobile Station (MS) [23]. It was observed that call dropping is not subjected to poor signal strength or failed handover alone. Our proposed model confirmed other causes of drop calls apart from low RxLev, handover and so on.

With this model, call drop rate could actually be reduced in GSM systems, hereby increasing the quality of service provided to subscribers by providing the basic minimum quality levels specified in [8], [9]. Furthermore, this model could be developed into an application that could be installed in mobile phone. This 
will take the reading of the different parameters and can detect the possibility of the on-going call dropping.

\section{CONCLUSION}

The use of ANN for the development of the call drop model can be used to make a classification and prediction of call drop events in GSM networks. The developed model has an accuracy of $87.5 \%$ prediction of drop call. The different causes of drop calls in the GSM system were understood. Call dropping is not subjected to poor signal strength or failed handover alone, but other network parameters made calls to drop, namely RxLev, RxQual, FER, BER and TA.

\section{ACKNOWLEDGEMENT}

This work is supported by the Department of Computer Engineering and Communications Engineering, Federal University of Technology, Minna, Nigeria, for providing adequate research laboratory.

\section{REFERENCES}

[1] A. S. Adegoke and I. T. Babalola, "Quality of Service Analysis of GSM Telephone System in Nigeria,” American Journal of Scientific Industrial Research, vol. 2, no. 5, pp. 707-712, 2011.

[2] J. O. Ajiboye, A. Tella, E. O. Adu, and J.I. Wojuola, "Stakeholders' Perceptions of the Impact of GSM on Nigeria Rural Economy: Implication for an Emerging Communication Industry," Journal of Community Informatics, vol. 3, no. 4, pp. 131-144, 2007.

[3] R. Jain, "Quality of Service (QoS) in Data Networks," Washington University in St. Louis, 2006. [Online]. Available: www.cse.wustl.edu. [Accessed January 20, 2016].

[4] Nigerian Communications Commission, “Subscriber Statistics Data," 2015. [Online]. Available: www.ncc.gov.ng/index.php?option= com_content\&view=article\&id=125:art-statisticssubscriber-data\&catid=65:cat-web-statistics\&Item id=73. [Accessed February 9, 2016]

[5] R. N. Ali, "Handoff and Drop Call Probability: A Case Study of Nigeria's Global System for Mobile Communications (GSM) Sector," Journal of Engineering and Technology, vol. 3, no. 2A, pp. 166-169, 2015.

[6] O. O. Roberts and K. E. Rowland, "Performance Evaluation of Service Quality of GSM Network Provider In Lagos, South-West Nigeria," International Journal of Innovative Research \& Development, vol. 1, no. 9, pp. 443 -451, 2010.

[7] G. Gu and G. Peng, "The Survey of GSM Wireless Communication System”, in International Conference on Computational Intelligence and Applications (ICCIA 2010), Tianjin, China, Dec. 2010, pp. 121-124.

[8] M.E Obota and P.E. Agbo, "Strategies for Improving Quality Of Service (QoS) of Global
System for Mobile Communication (GSM) in Nigeria," Journal of Research and Development, vol. 4, no. 1, pp 203-211, 2012.

[9] J. J. Popoola, I. O. Megbowon, and V.S. Adeloye, "Performance Evaluation and Improvement on Quality of Service of Global System for Mobile Communications in Nigeria," Journal of Information Technology Impact, vol. 9, no. 2, pp. 91-106, 2009.

[10] M. Pipikakis, "Evaluating and Improving the Quality of Service of Second-Generation Cellular Systems,” Bechtel Telecommunications Technical Journal, vol. 2, no.2, pp. 1-8, 2004.

[11] G. Boggia, P. Camarda, A. D’Alconzo, A. De Biasi, and M. Siviero, "Drop Call Probability in Established Cellular Networks: from Data Analysis to Modelling," in 2005 IEEE 61st Vehicular Technology Conference, Stockholm, Sweden, Jun. 2005, pp. 2775-2779.

[12] R. Zhao, X. Wen, D. Su and W. Zheng, "Call Dropping Probability of Next-Generation Wireless Cellular Networks with the Mobile Relay Station,” in 2010 Second International Conference on Future Networks, Hainan, China, Jan. 2010, pp. 63-67.

[13] Y. Iraqi and R. Boutaba "Handoff and Call Dropping Probabilities in Wireless Cellular Networks," in 2005 International Conference on Wireless Networks, Communications and Mobile Computing, Maui, USA, Jun. 2005, pp. 209-213.

[14] N. S. Tarkaa, J. M. Mom, and C.I. Ani, "Drop Call Probability Factors in Cellular Networks," International Journal of Scientific \& Engineering Research, vol. 2, no. 10, pp. 1-5, 2011.

[15] K. Sudhindra and V. Sridhar, "Root Cause Detection of Call Drops in Live GSM Network," in TENCON 2011 IEEE Region 10 Conference, Bali, Indonesia, Nov. 2011, pp. 25-33.

[16] Aster, “Aster Private Limited”, 2013. [Online] Available: http://aster.in/telecom/network_optimi zation.html [accessed December 20, 2015]

[17] A. R. Mishra, Advanced Cellular Network Planning and Optimisation. Wiley Publishing, 2006.

[18] Ascom, FER, RxQual, and DTX DL Rate Measurements in TEMS ${ }^{\mathrm{TM}}$ Investigation. UAE: Ascom, 2002.

[19] Round Solutions, 2016. [Online] Available: www.roundsolutions.com/pdf/gsmtester.pdf [accessed February 10, 2016]

[20] I. Ali, "Bit Error Rate (BER) Simulation Using MATLAB,” International Journal of Engineering Research and Applications, vol. 3 no. 1, pp. 706711, 2013.

[21] R. Chitranshi, J. Kushwaha, and P. Panchol. "Intelligent Optimization of GSM Network," International Journal of Engineering Science and Innovative Technology (IJESIT), vol. 1, no. 2, pp. 9-13, 2012. 
[22] M. Khosrowpour, Nanotechnology: Concepts, Methodologies, Tools, and Applications" Information Resources Management Association 1st ed. IGI Global, 2014.

[23] A. C. Messina, G. Caragea, P. T. Compta, F. H.P. Fitzek, and S. A. Rein, "Investigating Call Drops with Field Measurements on Commercial Mobile Phones," in 2013 IEEE 77th Vehicular Technology Conference (VTC Spring), Dresden, Germany, Jun. 2013, pp. 1-5. 